\title{
Traduire
}

Une autre perspective sur r tr traduction

Revue française de la traduction

$223 \mid 2010$

Tribunal et théâtre, faites entrer le traducteur

\section{Repenser la révision}

Défense et illustration de la relecture croisée

Jean-François Allain

\section{(2) OpenEdition}

Journals

Édition électronique

URL : http://journals.openedition.org/traduire/316

DOI : $10.4000 /$ traduire.316

ISSN : 2272-9992

Éditeur

Société française des traducteurs

Édition imprimée

Date de publication : 15 décembre 2010

Pagination : 114-120

ISSN : 0395-773X

Référence électronique

Jean-François Allain, «Repenser la révision », Traduire [En ligne], 223 | 2010, mis en ligne le 10 février 2014, consulté le 19 septembre 2020. URL : http://journals.openedition.org/traduire/316 ; DOI : https://doi.org/10.4000/traduire.316 


\section{REPENSER LA RÉVISION \\ Défense et illustration de la relecture croisée}

Jean-François ALLAIN

La situation d'un traducteur dans une organisation internationale (OI) présente, a priori, peu de points communs avec celle d'un traducteur en libéral. Sauf que tous deux exercent un même métier! Dans les pages qui suivent, Jean-François Allain rend compte d'une expérience menée au Conseil de l'Europe pour transformer la révision, traditionnellement conçue - ou vécue - dans les Ol comme une relation hiérarchique d'autorité et un exercice de "correction " parfois redouté, en un véritable travail de collaboration. Cette transformation des pratiques - et des mentalités - comporte une dimension psychologique (confiance en l'autre, confiance en soi), pédagogique (apprentissage mutuel) et professionnelle (gain de qualité) dont les enseignements sont transposables à d'autres situations et intéresseront notamment les traducteurs soucieux de sortir de leur " bulle".

\section{Introduction}

La " relecture croisée " est une méthode techniquement (mais pas forcément psychologiquement) assez simple à mettre en œuvre qui, pratiquée depuis plus du six ans dans le Service de la traduction française du Conseil de l'Europe, a eu des conséquences insoupçonnées au départ - au niveau tant individuel que collectif - en termes de formation, de motivation, de satisfaction au travail, d'homogénéité de la production et de qualité des relations humaines, sans doute parce que son principal mérite est d'intervenir sur le processus et non sur le produit.

En ce sens, elle est un élément essentiel dans la mise en place d'une politique d'assurance qualité.

Elle remplace avantageusement l'autorévision et, dans $80 \%$ des cas, rend inutile la révision au sens traditionnel du terme. 


\section{La révision traditionnelle}

La révision telle qu'elle est traditionnellement pratiquée dans les organisations internationales (nous n'entrerons pas ici dans le distinguo entre la révision d'un texte traduit par un seul traducteur et celle d'un texte partagé) a sans doute fait la preuve de son efficacité mais peut-être pas de son efficience. Autrement dit, les résultats obtenus (directs et indirects) ne sont pas forcément à la hauteur du temps qui lui est consacré ni du coût qu'elle entraîne. Globalement, elle présente en effet un ou plusieurs des inconvénients suivants :

- le réviseur contribue à améliorer le "produit " mais il n'a souvent pas le temps ni l'envie d'améliorer le producteur (le traducteur) ;

- le traducteur a souvent l'impression d'être en situation d'examen ; désireux de rendre la meilleure " copie " possible - pour autant que les délais le lui permettent -, il développe une crainte du " correcteur " qui l'inhibe plus qu'elle ne l'aide ;

- le traducteur est souvent perturbé quand il ignore qui le révisera ; il cherche donc à " intégrer " les manies des uns et des autres pour parer à toutes les critiques et évite de prendre des risques, condition nécessaire à toute amélioration ;

- l'absence d'explications sur la révision ne lui permet pas de faire la part entre corrections " nécessaires " et corrections " contingentes ", ni de tirer une leçon générale d'un cas d'espèce, n'ayant pas accès au raisonnement qui a motivé la correction ;

- le traducteur n'a pas droit à un " procès équitable ", en ce sens qu'il n'a pas la faculté de justifier ses choix ni de se justifier s'il se trouve qu'il a raison contre le réviseur ;

- il n'a pas l'occasion de progresser par réappropriation des bonnes idées du réviseur et d'intégrer certains réflexes dans ses schémas mentaux.

Pédagogiquement, donc, la révision traditionnelle est peu satisfaisante, même quand le réviseur prend le temps de faire des commentaires après coup. Elle favorise les relations de pouvoir topdown (de celui qui sait à celui qui ne sait pas) plutôt que de collaboration ; elle contribue peu à l'autonomisation du traducteur.

\section{La révision différenciée}

Le manque de temps et l'insuffisance des effectifs en interne obligent souvent les réviseurs à pratiquer une révision "différenciée " : révision attentive des parties sensibles ou difficiles (introduction, résumé analytique, décisions, recommandations), relecture rapide des parties factuelles (sans référence à l'original sauf en cas de doute), sondages aléatoires, etc. La compétence et l'expérience du traducteur (sa fiabilité) figurent aussi, naturellement, parmi les paramètres pris en compte dans le choix de la méthode à appliquer. 
Ce type de révision s'est développée depuis quelques années avec le recours accru à l'externalisation mais aussi, en interne, avec l'acceptation par les traducteurs et les réviseurs de la notion de qualité différenciée, c'est-à-dire d'une qualité qui peut être jugée "satisfaisante " ou " suffisante ", etc., compte tenu de la destination du texte, de sa criticité ou de sa technicité, du " cahier des charges » implicite ou explicite. Le texte à traduire n'est plus considéré comme une épreuve d'agrégation; la qualité n'est plus une Idée platonicienne.

Dans certains cas, trop rares, le réviseur peut, compte tenu de son expérience, donner des directives (choix terminologiques ou stylistiques, criticité du texte) avant que le traducteur ne commence son travail, ou donner des conseils en cours de traduction.

\section{La relecture croisée}

Le processus est simple : un traducteur lit sa traduction à haute voix à un collègue (traducteur ou réviseur) qui suit sur le texte original.

Selon la nature des textes, elle peut se pratiquer entre pairs ou dans un rapport plus hiérarchique, c'est-à-dire avec un traducteur chevronné, un réviseur ou un spécialiste du sujet traité. Elle peut éventuellement se faire avec un agent du service demandeur pour clarifier les éventuels points obscurs ou très techniques du texte original. Elle peut aussi être "différenciée ", c'est-à-dire porter sur la totalité ou sur partie seulement du texte.

NOTE : la relecture croisée peut aussi se faire avec une tierce personne (traducteur ou non) qui relit la traduction à haute voix tandis que le traducteur vérifie sur le texte original qu'il a bien rendu l'intégralité du sens. Une hésitation chez le lecteur est souvent le signe d'une maladresse de construction ou d'une ambiguité sémantique. De plus, certains défauts (des répétitions par exemple) s'entendent à l'oreille mieux qu'ils ne se voient.

Jusqu'ici, rien de bien spectaculaire en apparence, mais dès lors que la pratique dépasse le stade du " binôme " de deux personnes habituées à travailler ensemble pour se répandre à une grande partie d'un service et que le nombre de textes ainsi traités atteint une masse critique, la relecture croisée entraîne en cascade une suite d'avantages qui modifient à la fois l'ambiance et le mode de fonctionnement du service.

Naturellement, une telle opération suppose au départ l'instauration d'un climat de confiance entre les deux partenaires mais, inversement, une fois lancée, elle contribue fortement à développer un climat de collaboration confiante.

Concrètement, la méthode présente des avantages pour :

- le traducteur lecteur

- le traducteur/réviseur auditeur

- le service 
Pour bien les comprendre, il est important de rappeler deux handicaps majeurs du processus de traduction :

- la loi de la dilatation du temps de travail de Cyril Northcote Parkinson : "il faut autant de temps pour accomplir une tâche qu'on dispose de temps pour la faire ". Sauf contrainte de délai, le traducteur n'a " jamais fini " et a donc du mal à rendre son travail. II s'installe sur un " plateau " qui n'apporte aucun gain de qualité mais consomme du temps et génère parfois une angoisse obsessionnelle. La relecture croisée permet d'éviter ce risque si elle intervient dès l'arrivée sur le plateau, ou même (dans l'idéal) un peu avant. (Rappelons que les traducteurs ont, grâce aux outils dont ils disposent depuis quelques années, gagné du temps sur de nombreuses activités "périphériques" (temps de transmission, facilités de recherche documentaire, saisie et traitement du texte, etc.) ; en revanche, le temps de traduction per se semble " incompressible ». Or une analyse fine fait apparaître où sont les " pertes " sur ce plan et, par conséquent, où sont les gains possibles.)

- l'absence de repères objectifs de performance de qualité (étalonnage). Un coureur à pied peut se situer par rapport à ses concurrents (repères relatifs) ou par rapport aux records établis (repères absolus). Le jeune traducteur a du mal à "étalonner " la qualité de sa traduction. Au mieux, il " sent " si son travail est bon, mais ce sentiment n'a rien d'objectif ; il doit apprendre à " savoir " s'il est bon ou non, et à en identifier clairement les points faibles. La relecture croisée permet de progresser plus vite dans cette conscientisation et de mieux se " situer".

\section{Avantages pour le traducteur lecteur}

Par la pratique de la relecture croisée, le traducteur lecteur :

- accepte d'exposer ses faiblesses et ses doutes et d'entrer dans une relation de collaboration et de confiance où chacun participe solidairement à la qualité du travail de l'équipe ;

- constate les faiblesses et les doutes de ses partenaires de lecture et se rassure ainsi sur ses propres capacités (il constate par exemple que les passages qu'il a trouvé difficiles le sont aussi pour un collègue plus expérimenté) ; il prend confiance en soi, ce qui est un facteur de motivation et de productivité ;

- apprend plus vite les ficelles du métier par l'échange ou par les explications données en direct ;

- par la lecture à haute voix, soumet son texte à l'épreuve de ce que Flaubert appelait le "gueuloir " (" Je ne sais qu'une phrase est bonne qu'après l'avoir fait passer par mon gueuloir ") ; 
- sort d'un rapport à la révision hiérarchique et parfois traumatisant ; en face à face, le réviseur apparaît dans une situation d'explication plus que de jugement ; il se transforme en " coach";

- peut directement justifier des choix par rapport aux recherches qu'il a faites ou aux documents de référence qu'il a consultés ;

- apprend à faire la distinction entre corrections nécessaires et corrections contingentes, entre ses fautes (performance) et ses défauts (compétence) ;

- apprend à connaître ses points forts et ses points faibles et à mieux se situer sur l'échelle des compétences ;

- introduit de la variété dans sa routine quotidienne et une dimension sociale dans un travail par nature sédentaire et plutôt solitaire ;

- adopte plus rapidement une approche " conscientisée " de la traduction ; la discussion facilite la formalisation des processus traductionnels et est un facteur de stimulation intellectuelle.

\section{Avantages pour le traducteur auditeur (pair)}

La relecture permet à celui qui écoute la traduction et suit le texte original de :

- pratiquer un entraînement mental utile : comprendre rapidement le texte, comparer avec la traduction ;

- prendre connaissance d'un plus grand nombre de textes qu'il ne pourrait le faire normalement ;

- échanger sur des problèmes communs et partager des connaissances ;

- homogénéiser les textes si les deux partenaires travaillent sur un même sujet ;

- s'initier à la révision; il ne faut pas sous-estimer la capacité d'un jeune traducteur à faire preuve d'esprit critique et à proposer de bonnes solutions.

\section{Avantages pour le réviseur auditeur}

Celui-ci peut :

- transmettre en direct un savoir et une expérience sur les enjeux du texte, sur son contexte en général ou sur le fonctionnement de l'Organisation ; 
- faire passer le concept de "qualité suffisante ", par exemple en validant une traduction dont il juge la qualité " satisfaisante " et en expliquant qu'il est inutile de lui consacrer plus de temps ;

- transmettre les ficelles du métier en expliquant les raisons de ses choix ou de ses préférences ;

- aider le traducteur à anticiper les difficultés et à les résoudre plus facilement ;

- apprécier au passage les qualités du traducteur, en évitant de le juger et, encore moins, de le condamner ;

- guider graduellement la démarche d'apprentissage, notamment en sélectionnant les textes les mieux adaptés aux compétences d'un traducteur à tel ou tel stade de sa progression.

\section{Avantages pour le service}

Pour le service de la traduction considéré collectivement, la pratique de la relecture croisée présente deux grands avantages:

- elle accélère la transmission des savoirs et des savoir-faire au sein de l'équipe et garantit la pérennité du capital intellectuel ; corollairement, elle renforce l'homogénéité de la qualité de la production ;

- elle est un facteur indéniable de motivation et de satisfaction au travail, et a, de ce fait, des retombées sensibles sur la productivité.

\section{Gain de temps}

Une des objections préalables souvent invoquée contre la relecture croisée est le temps que nécessite l'exercice. Or, l'expérience montre que :

- dans le cas d'un simple contrôle qualité, la vitesse peut atteindre 2000 mots à l'heure. Même (ou surtout?) en situation d'urgence (équipes d'astreinte), deux traducteurs peuvent avoir intérêt à pratiquer ce travail à " quatre yeux " ;

- le traducteur fait oralement, avec son partenaire, une dernière lecture qu'il aurait faite de toute façon, généralement dans de moins bonnes conditions parce qu'il commence alors à " saturer "; de ce point de vue, on peut aussi concevoir l'exercice comme une autorévision mutuelle ;

- l'exercice permet de décider très vite du degré de révision dont un texte a besoin ; les textes qui exigent un travail de réécriture peuvent bifurquer vers une révision plus classique, mais 
une rapide lecture croisée aura déjà permis au réviseur de contrôler (plus facilement que par un contrôle "bord à bord ") la fidélité de la transcription du sens et d'identifier les parties à retravailler ;

- surtout, une bonne utilisation de la méthode permet de sortir de ce que l'on pourrait appeler un "syndrome de Pareto " (ou du principe du diminishing return), à savoir que le traducteur fait $80 \%$ du travail en $20 \%$ du temps, et qu'il s'installe ensuite sur une sorte de plateau coûteux en temps mais peu fructueux en gain de qualité.

\section{Conclusion}

II est sans doute peu souhaitable, voire contreproductif, de vouloir imposer la relecture croisée, mais la méthode peut, par l'exemple, faire tache d'huile en quelques années. On peut partir du principe qu'une partie de la population des traducteurs y restera de toute façon réfractaire, mais il est étonnant de voir avec quelle facilité beaucoup de jeunes l'adoptent, au point de ne plus pouvoir s'en passer. Sans doute parce que cette pratique répond à un désir profond et constant dans l'exercice de ce métier solitaire et sédentaire : le désir d'apprendre. Et, de fait, les adeptes de la méthode progressent plus vite.

Accessoirement, elle répond aussi au désir d'échanger (" sortir de sa coquille ") et elle renforce le sentiment d'appartenance à un " corps de métier ". Individuellement, les traducteurs ont davantage confiance en eux ; collectivement, ils ont une meilleure image d'eux-mêmes et une meilleure idée de la place qu'ils occupent dans l'ensemble de l'édifice. Enfin, ils acquièrent plus rapidement les savoirs liés au " cœur de métier".

Dans tous les cas, la relecture croisée occupe une place de choix dans une gestion de service qui entend répondre aux exigences de la TQM (Total Quality Management).

jean-francois.allain@coe.int

Jean-François ALLAIN est Chef du Service de la traduction française au Conseil de l'Europe et traducteur d'édition. 